\title{
Hommage - L'économie institutionnelle selon Martino Nieddu, lecteur du Temps des laboureurs...
}

\section{Gilles Allaire}

\section{Q OpenEdition}

1 Journals

Édition électronique

URL : http://journals.openedition.org/economierurale/6676

DOI : $10.4000 /$ economierurale.6676

ISSN : 2105-2581

Éditeur

Société Française d'Économie Rurale (SFER)

\section{Édition imprimée}

Date de publication : 30 juin 2019

Pagination : 7-17

ISSN : 0013-0559

\section{Référence électronique}

Gilles Allaire, « Hommage - L'économie institutionnelle selon Martino Nieddu, lecteur du Temps des laboureurs... », Économie rurale [En ligne], 368 | Avril-juin 2019, mis en ligne le 01 janvier 2021, consulté le 06 janvier 2021. URL : http://journals.openedition.org/economierurale/6676 ; DOI : https://doi.org/ 10.4000/economierurale.6676 


\title{
HOMMAGE
}

\section{L'économie institutionnelle selon Martino Nieddu, lecteur du Temps des laboureurs...}

\author{
Gilles ALLAIRE • US-ODR, INRA Toulouse, Auzeville
}

M artino Nieddu a apporté une solution originale et pertinente au traitement de la question agraire par la théorie de la régulation dès sa thèse (soutenue en 1997, sous la direction de Pierre Duharcourt), "Dynamiques de longue période dans l'agriculture productiviste et mutations du système agro-industriel français contemporain ", en soulignant I'hétérogénéité des "configurations productives" (coordinations autour d'un produit qui supportent une stratégie dans un « espace de concurrence ») ainsi que la variété et la permanence historique des logiques stratégiques des acteurs. Son apport s'est précisé avec sa « réévaluation du concept de patrimoine » (titre de son HDR). L'un des premiers parmi les économistes régulationnistes, avec Denis Barthélémy, il a mobilisé le cadre conceptuel de J.R. Commons (notamment le concept de « valeur raisonnable ») pour réduire la distinction entre « bien marchand » et « bien non marchand». Si Martino Nieddu a initialement abordé le patrimoine dans sa recherche d'une explication aux faits paradoxaux de l'agriculture du XXe siècle, ses travaux sur l'économie des services (avec Florence Gallois) et la chimie " verte " (avec Estelle Garnier, Nicolas Béfort, Franck-Dominique Vivien) montrent que cette notion a une portée bien plus large que les secteurs agricole et alimentaire'.

Enfin, Martino Nieddu a constamment soutenu que la méthode abductive (Pierce) était la façon correcte de raisonner pour expliquer les dynamiques sociales et il en a fait une pierre d'angle de sa conception de la Théorie de la régulation. Dans un texte court (Nieddu, 2013), paru dans la Revue de la Régulation, "Pourquoi lire Le Temps des laboureurs lorsqu'on est économiste, de surcroît régulationniste et travaillant sur les patrimoines économiques collectifs ? ", Martino Nieddu offre, sinon une synthèse, les points forts de sa position à la fois sur les approches régulationnistes et sur la question agraire. Soulignant l'originalité de l'analyse historique de la croissance des Xle-XIVe siècles, présentée par Mathieu Arnoux (2012), il vise à s'emparer " des enseignements contemporains de cet ouvrage ». Le présent texte, qui est un

1. Voir Barthélémy et al. (2003), Barthélémy et al. (2005), Barthélémy et Nieddu (2003), Barthélémy et Nieddu (2007), Gallois et Nieddu (2015), Nieddu et al. (2010), Nieddu et al. (2009), Nieddu et al. (2014), Nieddu et Vivien (2014), Nieddu (2005). 
hommage à Martino Nieddu, pourrait s'intituler : « Pourquoi lire Martino Nieddu lorsqu'on est économiste, de surcroît s'intéressant à la question de la terre et institutionnaliste ? ».

La lecture du livre de Mathieu Arnoux (2012), Le Temps des laboureurs : travail, ordre social et croissance en Europe (Xle-XIVe siècle), que nous propose Martino Nieddu (2013) - qui, rappelons-le, était historien de formation -, souligne que si on ne voit souvent que la raison économique dans l'émergence d'un cycle d'innovation ou une période de croissance, ce qui se passe de décisif est la production de ressources publiques et collectives indispensables à ce mouvement. Je développerai cinq points : 1) la lecture de Martino Nieddu est régulationniste ; 2) elle débouche sur un modèle général des " modernisations » agricoles; 3) une hypothèse abductive permet de penser le fait paradoxal qu'est la participation de la paysannerie à une logique d'accumulation capitaliste ; 4) la question agraire hier et aujourd'hui ; 5) pour conclure : extorsion versus développement.

\section{Passage par la théorie de la régulation}

Mathieu Arnoux mène une enquête, dans la seconde partie de son ouvrage, sur « une économie institutionnelle de l'Ordo Laboratorum ». Dans la première partie, intitulée " Histoires du laboureur ", il ne paraît " aucun paysan dont l'existence soit historiquement avérée » (Arnoux, 2012, p. 201), il s'agit plutôt de la place qui lui est accordée dans les justifications de la société d'ordre et dans la littérature. Aussi, l'auteur introduit la seconde partie en se démarquant des historiens du Moyen Âge pour qui «I'hypothèse que [la paysannerie] puisse contribuer de manière dynamique et innovante à la construction de l'économie et de la société n'est simplement pas envisagée " (Arnoux, 2012, p. 203). II s'attache, pour sa part, "à mettre en lumière trois séries d'institutions particulièrement importantes : les dîmes, les marchés et les moulins » (Arnoux, 2012, p. 219) et que l'on ne peut pas comprendre en occultant le rôle d'une paysannerie active.

Martino Nieddu tire des enseignements de sa lecture en tant que « régulationniste " pour deux raisons. Premièrement, les résultats que présente Mathieu Arnoux " posent la question plus générale de l'articulation entre la reconnaissance des statuts sociaux des hommes (et femmes) au travail, et les conditions de la réussite de transitions vers des régimes économiques de progrès (ici celui de la croissance des Xle-XIVe qui a desserré l'étau des contraintes matérielles sur les communautés rurales), et ce à un moment où l'articulation des problématiques de transitions énergétiques et de crise du capitalisme vient nous poser des questions de même nature » (Nieddu, 2013, p. 2). Ce faisant, il pointe les questions qui lui paraissent importantes pour notre communauté et, d'emblée, il ramène l'objet de l'analyse de M. Arnoux à la « construction de biens communs matériels et immatériels (dont une représentation idéologique de la société en trois ordres qui va contribuer à la croissance) » (Nieddu, 2013, p. 3).

Deuxièmement, Martino Nieddu reconnaît dans la façon de raisonner de Mathieu Arnoux la méthode de l'abduction². Les faits paradoxaux que cherche à contourner ou à expliquer

2. Pour sa part, il a affirmé cette posture méthodologique dès ses premiers travaux, en référence au pragmatisme de Pierce, en fixant quatre étapes à l'enquête scientifique (cf. Nieddu et Gaignette, 2000) : (1) la mise en ordre de faits stylisés paradoxaux ; (2) la mise en forme d'une hypothèse abductive, c'està-dire d'un principe général explicatif ; (3) la mise en œuvre d'une stratégie d'observation empirique de régularités étayant ce principe explicatif ; (4) un retour réinterprétatif pour s'assurer de la qualité heuristique de l'hypothèse. 
M. Arnoux tiennent d'une part à la méthode d'enquête dans un contexte où les sources écrites font défaut et d'autre part au fait historique que dans des économies féodales il y a pourraiton dire traces de la construction d'une autonomie des paysanneries, mais des traces indirectes. Le modèle explicatif vise à rendre compte de ce fait par l'hypothèse qu'une part du prélèvement sur le travail paysan, lui revient sous les formes de la solidarité et de l'investissement pour le collectif, ce que vérifient les enquêtes menées sur les dîmes et les droits de propriété afférents, le fonctionnement des marchés médiévaux et l'équipement hydraulique de l'Europe. Cette méthode pragmatique est également selon lui la façon de raisonner des régulationnistes (sans que nécessairement cela soit perçu ainsi par les chercheurs concernés).

\section{Un modèle général des « modernisations " agricoles}

Le modèle d'Arnoux est décrit par Martino Nieddu dans un langage régulationniste de la façon suivante : "Les conditions permissives de la croissance du Xle-XIII siècle [...] résident dans ce compromis institutionnalisé, arraché de façon aléatoire et toujours provisoire par la catégorie des laboratores, qui a su sortir de l'invisibilité sociale, pour s'imposer en tant que troisième ordre unifié par sa condition. La "révolution industrieuse" portée par cet ordre est soutenue par un ensemble de formes institutionnelles, qui vont se concrétiser dans trois types d'institutions, à la fois produits et productrices de patrimoines collectifs, que ce soit dans le domaine de la redistribution, de l'échange ou de la production: la dîme, le marché, et le moulin » (Nieddu, 2013, p. 4).

La croissance médiévale qui s'établit à la fin du $X^{e}$ siècle ne peut s'expliquer par une hypothèse technique et « la seule hypothèse convaincante est qu'elle résulta d'une augmentation massive et durable de l'offre de travail paysan... Le problème principal est alors d'expliquer une telle évolution, c'est-à-dire de comprendre ce qui poussa les habitants des campagnes à intensifier leurs efforts » (Arnoux, 2012, p. 15, in Nieddu, 2013, p. 4). Comme le souligne M. Nieddu : "Cette hypothèse d'une "révolution industrieuse" [...] suppose toutefois qu'on accepte dans le cas d'espèce que les paysans européens se sont constitués "en classe laborieuse, qui permit aux campagnes d'Europe de sortir de l'impasse d'une économie de subsistance" et que ceci "se fit en échange de compensations tangibles parce qu'elles étaient à la fois matérielles et symboliques" » (ibid.).

La notion de "révolution industrieuse » a été développée par Jan de Vries (1994) pour expliquer, dans I'Angleterre du XVII' siècle, le développement de la production et du marché des biens de consommation par l'augmentation de l'offre de travail salarié, sans changement de méthode ni innovation technologique majeure. M. Arnoux, qui expose en détail le débat des historiens sur l'existence et le rôle de la croissance démographique dans la croissance économique qui débute au $\mathrm{X}$ siècle en Europe, reprend cette hypothèse.

Les trois ordres, politique, religieux et du travail, amènent le troisième ordre à se constituer comme sujet autonome; si ce n'est pas le cas, le travail n'est que servitude. " [La] reconnaissance sociale accordée aux activités laborieuses dans une société où l'élite politique ou religieuse revendiquait l'exclusivité d'une vie oisive... fut l'une des conditions de l'accroissement de l'offre de travail, et donc de la croissance. " M. Nieddu souligne que le principe explicatif posé, qui réside dans le fait que la société d'ordres est pour les laboratores « revendication sociale et religieuse autant, voire plus, qu'obéissance à l'injonction providentielle énoncée par les clercs » (Arnoux, 2012, p. 343), est « perturbant en soi » mais peut devenir « consistant s'il permet effectivement de réinterpréter aussi les fondamentaux de la réalité du Moyen Âge ». 
Martino Nieddu montre que les analyses originales de Mathieu Arnoux de chacune des trois institutions étudiées convergent dans un modèle explicatif, auquel il donne une portée plus générale. II relève quatre fondamentaux de ce modèle, qui sans doute est un modèle des « modernisations " agricoles (pour peu que le travail de la terre soit effectué par des "paysans" ayant un minimum de libertés) ${ }^{3}$ :

- Une "volonté industrieuse ", motivée par la reconnaissance d'une position dans l'ordre social ; la reconnaissance d'un "ordre ", ou d'une "classe ", dans la globalité d'une société, est une forme de légitimation (qui n'en supprime pas la portée conflictuelle) de l'action collective par laquelle un « ordre » ou une « classe » prend existence et sur laquelle s'appuie la volonté industrieuse.

- Une " récompense " (rétribution) assurée par des mécanismes d'assurance et de redistribution (en l'occurrence la dîme et le marché médiéval considéré comme un espace protégé de l'arbitraire seigneurial).

- Une organisation collective (de classe) soutenant et pérennisant cette structure,

- Une source d'énergie, dont la gestion est collective et publique (les moulins à eau).

Les règles de marché sont complémentaires de la dîme et des institutions hospitalières comme instruments d'assistance aux pauvres. Cela est illustré, par exemple, dans le mécanisme suivant : la dîme est divisée en plusieurs parties, l'une sert à l'entretien du clergé et des seigneurs, mais une autre revient à la communauté, placée entre les mains des décimateurs, ressource dont l'usage est contraint par les « règles des marchés » (Arnoux, 2012, p. 252) : en temps normal, les céréales stockées sont vendues au prix courant des marchés, en temps de crise ou de disette, elles sont redistribuées par l'aumône. En même temps, ces mécanismes reproduisent la hiérarchie des ordres. Autrement dit, on ne peut séparer la question du développement des marchés durant la période de croissance médiévale d'une économie institutionnelle (globale) du travail. De la même façon, on ne peut séparer aujourd'hui la question des salaires ou des prix agricoles des politiques économiques et sociales, dont la politique agricole. Sur ce point, Martino Nieddu et Denis Barthélémy ont placé l'analyse des « aides » à l'agriculture dans le cadre d'une économie institutionnelle qui permet de comprendre comment la valeur de la production agricole résulte de transactions de différents types (Barthélémy et al., 2003 ; Barthélémy et Nieddu, 2007).

Le quatrième composant que souligne Martino Nieddu est capital. La croissance de l'offre de travail, dans des économies agraires, se traduit nécessairement : soit par une " faim de terre » (excédent de travail), qui peut se résorber par des défrichements ou des migrations, soit par l'intensification qui requiert plus de dépense d'énergie par ha (et pas seulement plus de travail). La thèse de la "révolution industrieuse " se traduit dans le monde des métiers, des arts et du commerce par des économies d'échelle essentiellement (mais elles vont toujours avec des innovations organisationnelles et dans les équipements...). S'agissant de la croissance agricole, en dehors des fronts pionniers, elle va avec certaines formes d'intensification. L'hypothèse ici avancée (à partir de laquelle Mathieu Arnoux interroge les travaux des historiens du Moyen Âge et généralisée dans la lecture de Martino Nieddu) est que les formes prises par cette intensification sont le résultat de l'action collective. Reste à poser la question de l'énergie mobilisable dans ce processus. Au Danemark, au XVIII siècle, par exemple, on assiste à une croissance agricole

3. Cette idée est développée dans Allaire (2019). 
alimentée par une révolution industrieuse qui se traduit par une croissance soutenue des productions animales; l'énergie nécessaire se trouvant dans les céréales importées.

Ce modèle restera à la base de la spécialisation industrielle du Danemark au XIX siècle, alimentant les grandes villes industrielles anglaises et allemandes ${ }^{4}$. Le « modèle des années 1960 " en France (Allaire, 1988) est aussi une forme de révolution industrieuse ; le livre de Debatisse (1963), leader du CNJA, La révolution silencieuse, en est un témoignage. En effet, les techniques et les savoirs qui ont permis alors la croissance agricole n'étaient pas en soi des innovations, les tracteurs étaient avant-guerre déjà nombreux dans le Nord de l'Europe ou les États-Unis, les principes de la fertilisation minérale depuis longtemps exposés, etc. L'innovation a été dans des changements culturels, sociaux et d'organisation du travail qui ont permis une appropriation massive de ces techniques, avant que n'intervienne plus tard une sophistication des techniques (et leur contestation). La source d'énergie pour cette forme d'intensification est le pétrole, la généralisation d'une intensification à l'américaine.

\section{Agriculture et logique d'accumulation capitaliste}

L'intégration capitaliste de l'agriculture est un fait paradoxal, dont la compréhension demande une hypothèse abductive. Sans le dire, ni même le savoir, c'est bien cette façon de raisonner qui m'a conduit à proposer un modèle de la croissance agricole française d'après-guerre (le « modèle de développement des années 1960 ») (Allaire, 1988) : une agriculture familiale et intensive (ou productiviste) participant à la croissance fordiste avec un régime d'accumulation " forcée » (thèse reprise par Martino Nieddu dans ces premiers travaux). Le XXe siècle est à fois celui de l'intégration de l'agriculture dans une logique d'accumulation capitaliste et celui du " triomphe de l'agriculture familiale ». Dans le contexte intellectuel des années 1980, mettre en lumière les institutions encadrant les investissements des agriculteurs, la qualification du travail productif, la sectorisation des marchés, et soutenant la croissance ainsi que la modernisation de l'agriculture, demandait d'abandonner les théories laissant la paysannerie à l'écart de l'accumulation capitaliste. Soit celle qui reconnaît la volonté industrieuse du paysan qui permet de nourrir les villes mais soutient que celui-ci s'exploite lui-même comme " petit producteur marchand " rognant son profit pour se maintenir en place ; soit la théorie dont le capital prélève le surtravail paysan sous la forme d'une extorsion. Dominé par le capital d'amont ou d'aval, le travail paysan devient hétéronome et l'agriculteur un travailleur surexploité de la banque. Chacune peut avoir sa part d'explication. Mais aucune ne peut rendre compte de la participation de l'agriculture et de la fraction moderniste de la paysannerie à un projet de développement capitaliste qui implique des formes de redistribution et la construction de patrimoines productifs collectifs. De la même façon que l'hypothèse d'une action collective ou "volontaire » de la paysannerie n'était pas envisageable pour les historiens du Moyen Âge qui étudiaient la construction de la société féodale, pour les courants marxistes dominant alors l'économie rurale, la participation « volontaire $»^{5}$ de la - petite et moyenne - paysannerie française à la croissance capitaliste et l'accumulation paysanne (de capital productif) n'était pas pensable.

Au contraire, l'hypothèse abductive (et régulationniste) consistait à expliquer l'accumulation dans le secteur agricole et l'inscription de l'agriculture dans le cadre du mode de développement fordiste en spécifiant le jeu des cinq « formes institutionnelles » canoniques de l'approche

4. Pour un développement de cet exemple, voir Allaire (2019).

5. Pour reprendre le terme de M. Arnoux. 
régulationniste ${ }^{6}$ dans la croissance de ce secteur ; la vérification de la cohérence de ce modèle explicatif passant par l'analyse de la variété des trajectoires selon les filières. Pour sa part - et c'est un apport décisif à la compréhension de cette période -, M. Nieddu a proposé de rejeter la thèse "d'une configuration productive unique de l'agriculture du fordisme », pour considérer " deux agricultures productivistes », " deux configurations qui se reproduisent dans le temps ». Celle des grandes exploitations qui avaient échoué dans les décennies précédentes à concentrer la terre du fait de la résistance d'une paysannerie semi-prolétaire et qui trouvaient un nouvel espace économique ; ces " gros paysans » se suffisaient d'une régulation sectorielle qui protège du spectre de la subordination par le capital agro-alimentaire réduite à l'organisation du marché. Celle des exploitations moyennes en croissance dans un contexte de crédit bon marché, qui se présente de façon beaucoup plus paradoxale ; pour soutenir leur croissance rapide, celles-ci ont besoin d'une alliance avec un capital alimentaire qui assure l'écoulement de leur production, ce qui passera par le développement des coopératives et de patrimoines professionnels collectifs. II introduit ainsi une distinction entre des projets « productivistes », que l'on peut appeler libéraux et « modernistes » (néo-corporatistes). Pour souligner son propos, il affirme que c'est entre ces deux projets qu'il faut chercher les « compromis institutionnalisés » à la base de la croissance agricole de la période (Nieddu, 1999) ${ }^{7}$.

Cette analyse pose la question du statut du modèle par rapport à l'histoire dans sa complexité. Sans doute peut-on parler d'une " agriculture du fordisme » pour en définir des traits globaux (l'affirmation sociale d'une paysannerie familiale entrepreneuse et sa reconnaissance par un statut et des droits sociaux, l'intensification capitalistique des productions végétales et animales, la centralisation bancaire des crédits pour l'investissement, le développement de la formation professionnelle et la construction de patrimoines coopératifs matériels et immatériels) mais il ne s'agit pas d'un modèle unique d'organisation qui éliminerait la variété des « configurations productives » et des projets politiques, les " compromis institutionnalisés " s'inscrivent dans une grande variété de " blocs sociaux régionaux " (Lipietz, 1983) si l'on étudie l'agriculture française ou les agricultures de l'Europe de l'Ouest dans les sociétés d'après-guerre. De même, le modèle d'Arnoux est explicatif de la croissance médiévale, mais il n'y a pas « une " paysannerie ou une agriculture européenne. Se pose alors la question du rapport des approches régulationnistes (de leurs modèles explicatifs) à l'histoire, qui a beaucoup intéressé Martino Nieddu comme cela transparaît dans plusieurs textes, dont celui-ci.

\section{La question agraire, hier et aujourd'hui}

De la même façon que l'on ne peut comprendre les politiques agricoles modernes comme un simple instrument d'extorsion du surtravail paysan, on ne peut les comprendre comme simple support de la base sociale d'accumulation qu'elles délimitent, comme s'il s'agissait d'une transition instantanée. II s'agit de politiques ayant une dimension sociale, distributive. Le projet des années 1960 repose sur la «thèse des trois agricultures " (déjà compétitive, à moderniser,

6. Il s'agit de l'État (sous la forme de l'État moderne avec ses politiques sectorielles et de l'Étatprovidence avec le système de protection sociale), de la monnaie (en l'occurrence la monnaie de crédit), des formes de concurrence (et de coopération), du régime international (en l'occurrence du GATT qui laisse possible des politiques agricoles nationales) et du « rapport salarial » qui ne concerne pas l'agriculture en tant que forme de travail mais indirectement avec le développement de la « société salariale ». 7. C'est l'enquête sur la variété des configurations productives au sein de configurations territoriales et de blocs sociaux régionaux qui a conduit Martino Nieddu à s'inscrire dans une approche «mésoéconomique » (Lamarche et al., 2015). 
à éliminer) pour lesquelles sont fixés des objectifs spécifiques de politiques publiques. S'agit-il de politiques séparées ? Non. D’une part, elles sont simultanément défendues par le syndicat majoritaire (où sont représentés des intérêts variés) et, d'autre part, elles dépendent du même domaine de l'action publique (le ministère de l'Agriculture, qui, rappelons-le, gère la politique économique, la protection sociale, l'enseignement agricole et la recherche...). Sur le plan théorique, pour expliquer simultanément les dynamiques en cause, il faut concevoir un principe général qui agit dans les transactions, qu'elles soient (au sens de Commons) marchandes, managériales ou de répartition (des bénéfices de la croissance), à côté de ce que serait une pure rationalité économique calculatoire.

Martino Nieddu (avec Denis Barthélémy, Franck-Dominique Vivien, Pascal Grouiez et d'autres) a recherché ce principe dans les concepts de "valeur raisonnable " (Commons), de " biens identitaires", de "relations patrimoniales » et de " patrimoines productifs ». Comme les constructions théoriques que j'ai pu faire de mon côté, en m'appuyant aussi sur les concepts commonsiens de "dettes d'autorité " et de " propriété intangible » (e.g., Allaire, 2017), ce sont des travaux qui restent encore exploratoires et renvoient à une vaste question. Je dirais qu'il faut encore monter en compréhension, en continuant de développer des analyses empiriques dans différents champs, périodes et contextes. C'est pour cela qu'il convient d'apprécier à leur juste mesure les fulgurances synthétiques de Martino Nieddu quand il nous invite à lire Le Temps des laboureurs...

Si l'on voulait définir en deux mots la « régulation » (celle de la théorie de la régulation), on pourrait dire qu'il s'agit de la construction sociale des politiques et des patrimoines collectifs. Pour reprendre les termes de M. Nieddu, il s'agit de la « construction des biens publics et des patrimoines collectifs ». Cette formule introduit une hiérarchie (et une division fonctionnelle) dans la variété des "transactions de répartition " (répartir, par exemple, les bénéfices du progrès génétique, la rente foncière en Russie ou les aides agricoles européennes, sont des problèmes différents, mais de même nature). Quoique le terme « bien » soit embarrassant (mais utile pour parler à des économistes), la distinction que fait M. Nieddu renvoie (dans mes propres termes) à deux formes de la propriété intangible, (i) la " propriété sociale » (Castel, 2008), c'est-à-dire les droits et statuts créés par les politiques économiques et sociales, qui sont garantis par une « dette sociale » (Théret, 2013) et dépendent des rapports sociaux; (ii) les patrimoines productifs, considérés comme des systèmes complexes de ressources communes (au sens d'Ostrom), qui relèvent d'une propriété collective et délimitent une grande diversité de configurations productives et de "biens communs ". L'articulation entre ces deux dimensions dépend des contextes sociohistoriques.

Quels sont les principes souverains qui dans le champ de l'agriculture ou dans le champ de l'alimentation justifient des droits et la fixation des prix ? Au temps des laboureurs, la menace essentielle est celle des pénuries alimentaires, elle justifie des politiques frumentaires. La question de la sécurité alimentaire reste au cœur des politiques agricoles modernes (XXe siècle), sous la forme de l'intégration à des schémas (nationaux) d'accumulation du capital et d'une régulation des revenus agricoles, dans un contexte où ne cesse de s'accroître la population urbaine et où l'industrialisation (utilisation des énergies fossiles) a permis aux sociétés industrielles d'échapper à la loi de Malthus. Ce qui est alors propre aux projets de modernisation agricole et aux périodes de croissance, pour le monde " occidental » au moins, depuis le Xle siècle, jusqu'à la Politique agricole commune de l'Europe, en passant par le New Deal aux États-Unis, c'est le rapprochement entre sécurité alimentaire et « agriculture familiale » (ou propriété paysanne), mais dans des configurations politiques et sociales fort différentes. 
La crise de l'agriculture productiviste que les régulationnistes ont analysée depuis les années 1990 (Allaire et Boyer, 1995) est aujourd'hui actée, les politiques agricoles sont désormais dites "politiques de transition », et l'on pourrait même dire qu'elle est dépassée... par la refondation du projet moderniste; mais refondation ambivalente entre la fuite en avant dans un néo-productivisme et la refondation des patrimoines collectifs. À cet égard, il conviendrait d'étudier, depuis les années 1990, les transformations et la recomposition des projets libéraux et modernistes qu'avait identifiés Martino Nieddu, ainsi que les évolutions et les conflits autour des patrimoines collectifs agricoles ${ }^{8}$.

La conscience des limites écologiques et de la gravité des dégâts résultant de l'industrialisation a fait de la crise de l'agriculture une crise structurelle radicale. Elle se traduit par des changements dans les marchés où s'affrontent des logiques différentes d'acteurs, dans la remise en question de la délimitation et des objectifs des politiques agricoles. Ce qui fait tenir les politiques agricoles, c'est leur caractère de "dette sociale ", mais en même temps elles se sont départies d'une partie de leurs objectifs sociaux. II faut sans doute changer radicalement de paradigme pour voir l'avenir de l'agriculture au-delà du productivisme et imaginer des rapports « raisonnables » des sociétés, urbaines comme rurales, à la terre et à l'alimentation. Cette question est très présente dans les derniers travaux de Martino Nieddu et son intérêt pour l'économie écologique. On la retrouve avec le rôle clé des moulins dans le modèle de croissance du Temps des laboureurs. Elle l'était aussi dans nos échanges, inachevés, autour de la Part maudite de Georges Bataille...

\section{Extorsion versus développement}

Lire Le Temps des Laboureurs enseigne que l'on ne peut penser l'histoire des sociétés et les rapports de classes du point de vue limité de l'extorsion, qui certes est toujours une tentation pour les puissants, qu'il s'agisse des autorités religieuses ou seigneuriales médiévales ou des classes capitalistes. II ne s'agit pas de nier l'exploitation, mais de reconnaître que la socialisation du surtravail finance non seulement l'accumulation du capital et l'existence des autorités mais aussi l'action publique et la construction de patrimoines collectifs.

Martino Nieddu, dans sa lecture du Temps des laboureurs, met en avant deux caractéristiques du modèle de croissance de ce temps qui peuvent être réinvesties pour l'analyse des modernisations des siècles suivants jusqu'au $X X^{\top}$ siècle. Premièrement, le prélèvement sur le travail, quelle qu'en soit l'autorité qui en est l'auteur, n'est pas uniquement un détournement de l'agriculture, il est l'instrument de la « protection au sein des communautés au travail » et de la production de « services publics ». Deuxièmement la production de " services publics » est aussi « le résultat d'un engagement collectif, et s'inscrit dans une construction économique plus globale, celle des biens collectifs ou communs nécessaires à l'existence dans une économie substantive ».

II souligne une double difficulté pour élargir cette approche à d'autres périodes, qui est que l'existence des biens collectifs ou communs et plus précisément des patrimoines collectifs productifs n'est pas perçue et étudiée à sa juste place. " Les arrangements économiques, les stratégies de coopération entre acteurs par ailleurs concurrents, les accords de limitation de la concurrence, les dispositifs de distribution ordonnée de la connaissance et des revenus, les garanties de stabilité de l'emploi qui préservent les compétences, etc., cet ensemble de dispositifs collectifs qui permettent la production et sont au cœur d'une économie substantive échappent alors à

8. Sur le cas de la sélection génétique animale, voir Allaire et al. (2018).

14 • Économie Rurale 368/Avril-Juin 2019 
la fois à l'analyse et aux préconisations. » De plus, la construction et la défense des patrimoines collectifs demandent de construire " des discours de légitimation d'actions "non naturelles" dans le contexte des rapports de forces sociaux, et qui, en cherchant à se frayer un passage dans celui-ci, doivent toujours leur payer un tribut ${ }^{9}$. Les rapports sociaux sont en effet des rapports de pouvoir et, si des choix « raisonnables » peuvent émerger de conflits, ils n'éteignent pas cette conflictualité. C'est en ce sens que les justifications de ces choix sont « non naturelles ».

Martino Nieddu termine sa lecture du Temps des laboureurs en annonçant une " mauvaise nouvelle » : "Si l'on raisonne en longue période, une telle séquence ["révolution industrieuse" I compromis institutionnalisé construisant des mécanismes de prélèvement, de protection et de redistribution / cercle vertueux de croissance] semble se trouver derrière nous, dans les conflits qui avaient amené les couches laborieuses industrielles à se constituer en "classe pour soi" et à arracher à la fois un statut salarial et des mécanismes de redistribution assurant l'accès à la consommation. » Aujourd'hui, on se trouverait plutôt « dans un type de période confiscatoire des fruits du travail et destructrice de solidarités ». Est-on à la fin d'un long cycle de développement (du temps des laboureurs à celui de l'agriculture familiale intensive du XXe siècle) ? Cette opinion ouvre de nouveau une grande question pour les régulationnistes, sur laquelle Martino aurait continué de réfléchir !

Ce que je voudrais souligner pour conclure, c'est qu'il liait cette question à celle des « enjeux des grandes transitions énergétiques nécessaires aujourd'hui ", que l'on devrait réfléchir, avec l'exemple du travail de Mathieu Arnoux, " à l'aune de l'expérience de la transition décentralisée réussie et de la production de patrimoines collectifs par les communautés paysannes, malgré et au-delà de la violence des tentatives d'accaparement féodaux. "

Ce texte est une version complétée du texte présenté au Congrès de l'AFEP, à Reims, lors de la table ronde : Hommage à Martino Nieddu, le 6 juillet 2018.

9. Ici, tribut doit sans doute se comprendre en plusieurs sens. 


\section{RÉFÉRENCES BIBLIOGRAPHIQUES}

Allaire G. (1988). Le modèle de développement agricole des années 1960. Économie rurale, $\mathrm{n}^{\circ} 184-185-186$, pp. 171-181.

Allaire G. (2017). L'ambivalence de la socialisation de l'agriculture. In Allaire G., Daviron B. (dir.), Transformations dans l'agriculture et l'agro-alimentaire. Entre écologie et capitalisme. Versailles, Quae, coll. « Synthèse », pp. 359-378.

Allaire G. (2019, à paraître). Ambivalence, modèles et paradoxes de la modernisation de l'agriculture. In Christophe Bonneuil, Léna Humbert, Margot Lyautey, Une autre histoire des modernisations agricoles au XX $X^{e}$ siècle. Rennes, Presses universitaires de Rennes.

Allaire G., Boyer R. (dir.) (1995). La grande transformation de l'agriculture. Lectures conventionnalistes et régulationnistes. Paris, Inra/Economica.

Arnoux M. (2012). Le Temps des laboureurs : travail, ordre social et croissance en Europe $\left(X I^{e}\right.$-XIV siècle). Paris, Albin Michel, coll. «L'Évolution de l'humanité ».

Barthélémy D., Boinon J.-P., Nieddu M. (2003). Le dualisme comportemental des agriculteurs : une interprétation institutionnaliste dialectique. Économie et institutions, $n^{\circ} 3,2^{\mathrm{e}}$ semestre, pp. 118-150.

Barthélémy D., Nieddu M. (2003). Multifonctionnalité agricole, biens marchands ou biens identitaires. Économie rurale, $\mathrm{n}^{\circ}$ 273-274, pp. 103-120.

Barthélémy D., Nieddu M. (2007). Non-trade concerns in agricultural and environmental economics: How JR Commons and Karl Polanyi can help us. Journal of Economic Issues, vol. 41, $\mathrm{n}^{\circ}$ 2, pp. 519-527.

Barthélemy D., Nieddu M., Vivien F.-D. (2005). Économie patrimoniale, identité et marché. In Barrère C., Barthélemy D., Nieddu M., Vivien F.-D. (dir.) (2005). Réinventer le patrimoine. De la culture à l'économie: une nouvelle pensée du patrimoine ? Paris, L'Harmattan, pp. 121-150.

Castel R. (2008). La propriété sociale : émergence, transformations et remise en cause. Esprit, ${ }^{\circ}$ 8, pp. 171-190.
Debatisse M. (1963). La révolution silencieuse. Paris, Calmann-Lévy, 275 p.

Gallois F., Nieddu M. (2015). Quand l'État décrète le marché : le cas du Plan Borloo. Revue de la régulation, $\mathrm{n}^{\circ} 17,1^{\mathrm{er}}$ semestre, $\mathrm{http}: / /$ regulation.revues.org/11209.

Lamarche T., Nieddu M., Grouiez P., Chanteau J.-P., Labrousse A., Michel S., Vercueil J. (2015). Les régulations mésoéconomiques : saisir la variété des espaces de régulation. In La théorie de la régulation à l'épreuve des crises, Paris, pp. 1-23.

Lipietz A. (1983). Le Capital et son espace (deuxième édition augmentée). Paris, La Découverte ( $1^{\text {re }}$ édition, Paris, Maspero, 1977).

Nieddu M. (1999). Secteurs et territoires, tour à tour problèmes et solutions dans les approches régulationnistes. Bilan de travaux récents et application à la dynamique de l'agriculture française des trente glorieuses. Séminaire Régulations Sectorielle et Territoriale.

Nieddu M. (2005). Capitalisme et patrimoine ? In Barrère C., Barthélemy D., Nieddu M., Vivien F.-D. (dir.) (2005), Réinventer le patrimoine. De la culture à l'économie: une nouvelle pensée du patrimoine? Paris, L'Harmattan, pp. 151-177.

Nieddu M. (2007). Le patrimoine comme relation économique. Économie appliquée, vol. $60, \mathrm{n}^{\circ} 3$, pp. 31-55.

Nieddu M. (2007). Revisiter l'économie du service à partir de la théorie économique du patrimoine. In Heurgon E., Landrieu J. (dir.), L'économie des services pour un développement durable. Nouvelles richesses, nouvelles solidarités. Paris, L'Harmattan.

Nieddu M. (2013). Pourquoi lire Le Temps des laboureurs lorsqu'on est économiste, de surcroît régulationniste et travaillant sur les patrimoines économiques collectifs ? Revue de la régulation [En ligne], $\mathrm{n}^{\circ} 14$.

Nieddu M., Gaignette A. (2000). L'agriculture française entre logiques sectorielles et logiques territoriales (1960-1985). Cahiers 
d'économie et de sociologie rurale, $\mathrm{n}^{\circ}$ 54, Nieddu M., Vivien F.-D. (2014). Quels apports pp. 49-87.

de l'approche patrimoniale pour l'ana-

Nieddu M., Garnier E., Bliard C. (2010). L'émergence d'une chimie doublement verte. Revue d'économie industrielle, $\mathrm{n}^{\circ} 132$, pp. 53-84. lyse des transitions vers le développement soutenable: l'exemple de la chimie verte. Économie appliquée, vol. 67, $\mathrm{n}^{\circ} 4$, pp. 40-70.

Nieddu M., Garnier E., Bliard C. (2014). Patrimoines productifs collectifs versus exploration/exploitation. Revue économique, vol. 65, n 6 , pp. 957-987.

Nieddu M., Petit O., Vivien F. D. (2009). Identités, patrimoines collectifs et développement soutenable. Développement durable et territoires. Économie, géographie, poliNieddu M., Vivien F. D., Garnier E., Bliard C. (2014). Existe-t-il réellement un nouveau paradigme de la chimie verte? Natures Sciences Sociétés, $\mathrm{n}^{\circ} 22$, pp. 103-113.

Théret B. (2013). Dettes et crise de confiance dans l'euro: analyse et voies possibles de sortie par le haut. Revue française de socioéconomie, $\mathrm{n}^{\circ} 12$, pp. 91-124. 